\title{
Plasma protein supplements modulate the activation of gut-associated immune system induced by Staphylococcus aureus enterotoxin B in rats
}

\author{
A. Pérez-Bosque ${ }^{1}$, L. L. Miró ${ }^{1}$, J. Polo $^{2}$, L. Russell $^{3}$, J. Campbell $^{3}$, E. Weaver $^{3}$, J. Crenshaw $^{3}$ \\ and M. Moretó ${ }^{1}$ \\ ${ }^{1}$ Departament de Fisiologia, Facultat de Farmàcia, Universitat de Barcelona, Spain, ${ }^{2}$ APC Europe, Granollers, \\ Spain and ${ }^{3}$ APC Inc, Ankeny, IA 50021, USA
}

\begin{abstract}
Supplementation of diets with plasma protein has been shown to prevent the activation of lymphocyte populations of Peyer's patches and mesenteric lymph nodes ${ }^{(1)}$ and improve the intestinal epithelial barrier function in a rat model of intestinal inflammation ${ }^{(2)}$. The present study investigated the effects of porcine plasma proteins (SDAP) and Ig concentrate (IC) supplements on diffuse gut-associated lymphoid tissue in a model of mild intestinal inflammation. The different populations of lamina propria and intraepithelial lymphocytes, as well as mucosal expression of cytokines (interferon- $\gamma$ (IFN- $\gamma$ ), TNF $\alpha$, IL-6 and IL-10) and pro-inflammatory mediators (inducible NO synthase (iNOS) and leukotriene $\left.\mathrm{B}_{4}\left(\mathrm{LTB}_{4}\right)\right)$, were investigated. Wistar-Lewis rats were fed diets supplemented with SDAP (80 g/kg; $n$ 9), IC (15 g/ $\mathrm{kg} ; n$ ) or milk proteins (control die; $n$ 9) from weaning (day 21) to day 33 or 34 after birth. On days 30 and 33 rats were administered $S$. aureus enterotoxin B (SEB; $0.5 \mathrm{mg} / \mathrm{kg})$. Experimental groups were control, SEB, SEB-SDAP and SEB-IC. Lymphocyte populations were analysed by immunohistochemistry on day 34 (i.e. $24 \mathrm{~h}$ after SEB administration). The markers used were: CD3 (T lymphocytes), CD25 (activated T lymphocytes), CD4 (T-helper lymphocytes), CD8 (T-suppressor/cytotoxic lymphocytes), TCR $\gamma \delta$ (T- $\gamma \delta$ lymphocytes) and NKPR1A (NK cells). Cytokines were determined by a cytometric bead array assay, $\mathrm{LTB}_{4}$ by commercial ELISA and iNOS by real-time PCR in mucosal homogenates, all at $6 \mathrm{~h}$ after SEB administration.

In both lamina propria and epithelium compartments SEB increased the lymphocyte cytotoxic populations (T- $\gamma \delta 40 \%$ and $70 \%$; NK cells $60 \%$ and $75 \%$ respectively, all $P<0.05)$ and doubled the number of activated T lymphocytes $(P<0.001)$. Both SDAP and IC prevented the SEB effects on the lamina propria, while in the epithelium only SDAP reduced the extent of T-cell activation $(P<0.05)$. SEB increased mucosal iNOS expression by $28 \%(P<0.05)$ and both plasma protein supplements prevented SEB effects on iNOS expression in the intestinal mucosa (both $P<0.05$ ).

In the mucosa SEB doubled IFN- $\gamma$ and $\mathrm{LTB}_{4}$ concentrations and increased TNF $\alpha$ and IL- 6 concentrations by $\left.20-30 \% ; P<0.05\right)$. SDAP partially prevented these effects on IFN- $\gamma$, IL-6 and $\mathrm{LTB}_{4}(P<0.05)$. IC was also effective in reducing the expression of TNF $\alpha$ and $\mathrm{LTB}_{4}$ in the mucosa $(P<0.05)$. It is concluded that dietary supplementation with plasma proteins can attenuate the intestinal inflammatory effects induced by SEB.
\end{abstract}

1. Pérez-Bosque A, Pelegrí C, Vicario M et al. (2004) J Nutr 134, 2667-2672.

2. Pérez-Bosque A, Amat C, Polo J, Campbell JM, Crenshaw J, Russell L \& Moretó M (2006) J Nutr 136, $2838-2843$. 